\title{
3D surface-related multiple prediction: A sparse inversion approach
}

\author{
E. J. van Dedem ${ }^{1}$ and D. J. Verschuur ${ }^{2}$
}

\begin{abstract}
The theory of iterative surface-related multiple elimination holds for $2 \mathrm{D}$ as well as $3 \mathrm{D}$ wavefields. The $3 \mathrm{D}$ prediction of surface multiples, however, requires a dense and extended distribution of sources and receivers at the surface. Since current 3D marine acquisition geometries are very sparsely sampled in the crossline direction, the direct Fresnel summation of the multiple contributions, calculated for those surface positions at which a source and a receiver are present, cannot be applied without introducing severe aliasing effects. In this newly proposed method, the regular Fresnel summation is applied to the contributions in the densely sampled inline direction, but the crossline Fresnel summation is replaced with a sparse parametric inversion. With this procedure, $3 \mathrm{D}$ multiples can be predicted using the available input data. The proposed method is demonstrated on a 3D synthetic data set as well as on a 3D marine data set from offshore Norway.
\end{abstract}

\section{INTRODUCTION}

Following Weglein (1999) multiple-attenuation techniques can be divided into methods that exploit differences in spatial and temporal properties between primary and multiple reflection events, e.g., moveout filtering techniques, and methods that are based on the wave equation, e.g., multiple prediction followed by subtraction. The surface-related multipleelimination (SRME) method (Verschuur, 1991) belongs to the wave equation methods and is an interface-related approach; therefore, it removes all multiples related to that interface, in this case the surface. Moreover, the method requires no information about the subsurface, only about the surface, e.g., the surface reflectivity and the source and receiver characteristics.
Although the SRME method applies to 2D as well as 3D wavefields (as demonstrated by Verschuur, 1991), the current implementation is limited to application to 2D data sets, since the most important prerequisite for $3 \mathrm{D}$ data sets (complete and dense sampling of sources and receivers along both spatial axes) is not met. In most practical situations, however, primary and multiple reflections have crossline components, either because the data are acquired along two spatial coordinates, or because of crossline structure in the subsurface, or both. Application of the 2D SRME method in these situations results in amplitude and traveltime errors in the predicted multiple reflections.

For 3D data acquired over a complex 3D subsurface, surface-related multiples should be predicted in a true $3 \mathrm{D}$ sense, taking into account 3D propagation of the multiple wavefield. Although 3D surface-related multiple prediction is possible in theory, practical and economical constraints prevent its straightforward application and forces one to seek a pragmatic solution. Current research on 3D multiple attenuation can be divided into two approaches: true 3D surfacerelated multiple prediction on one hand, which is the focus of this article, and more flexible subtraction of 2D predicted multiples on the other (see, e.g., Ross et al., 1999; Ikelle and Yoo, 2000).

Application of 3D surface-related multiple prediction to $3 \mathrm{D}$ data sets requires the source and receiver wavefield to be densely sampled with sufficient aperture along the two spatial coordinates. Because these sampling conditions are never met using standard marine or land-acquisition geometries, undersampling of the wavefield has to be compensated for either prior to 3D surface-related multiple prediction, defined as global data reconstruction (interpolation and/or extrapolation), or during 3D surface-related multiple prediction, defined as Fresnel zone reconstruction. Global data reconstruction aims at reconstructing the data necessary for application of 3D surface-related multiple prediction. Several data reconstruction methods with different levels of sophistication

\footnotetext{
Manuscript received by the Editor July 28, 2003; revised manuscript received May 12, 2004; published online May 23, 2005.

${ }^{1}$ Formerly Delft University of Technology, P.O. Box 5046, 2600 GA Delft, The Netherlands; presently Shell International Exploration and Production B.V., Kessler Park 1, 2288 GS Rijswijk, The Netherlands. E-mail: Ewoud.vanDedem@shell.com.

${ }^{2}$ Delft University of Technology, Faculty of Applied Sciences, P.O. Box 5046, 2600 GA Delft, The Netherlands. E-mail: d.j. verschuur@tnw.tudelft.nl.

(C) 2005 Society of Exploration Geophysicists. All rights reserved.
} 
have been proposed for enabling 3D surface-related multiple prediction (see, e.g., Nekut, 1998; Matson and Corrigan, 2000; Biersteker, 2001; van Dedem, 2002).

Instead of interpolating data prior to 3D multiple prediction for 3D marine data sets, Sun (1999) proposes the use of available 3D data (under the assumption that seismic traces within one CMP gather are invariant with azimuth) to arrive at an approximate intermediate 3D multiple prediction result, which is then interpolated to obtain an alias-free 3D multiple prediction result. Then, the alias-free 3D multiple prediction result is retrieved by reconstructing the Fresnel zones at the surface of the multiple wavefield through a parametric sparse inversion of the (sparsely sampled) crossline multiple contributions. In the context of this article, the Fresnel zone refers to the part of the seismic wavefield that, after the inline multiple prediction process, actually contributes to the prediction of the surface-related multiple by the crossline summation. In the next section, the iterative surfacerelated multiple prediction and elimination theory is briefly reviewed.

\section{ITERATIVE SURFACE-RELATED MULTIPLE ELIMINATION}

For formulation of surface-related multiple elimination (SRME), the matrix notation for seismic wavefields introduced by Berkhout in 1982 is used.

\section{Review of iterative surface-related multiple elimination}

Following Berkhout and Verschuur (1997), the multiplefree upgoing wavefield $\mathbf{P}_{0}^{-}(\omega)$ can be estimated using a Neumann series expansion from the data with multiples. The recursive relation is expressed as

$$
\mathbf{P}_{0}^{-}(\omega)^{(n+1)}=\mathbf{P}^{-}(\omega)-A(\omega) \mathbf{P}_{0}^{-}(\omega)^{(n)} \mathbf{P}^{-}(\omega),
$$

with $n$ being the recursion number. The data containing surface-related multiples $\mathbf{P}^{-}(\omega)$ can be taken as the initial estimate of the multiple prediction operator, i.e.,

$$
\mathbf{P}_{0}^{-}(\omega)^{(0)}=\mathbf{P}^{-}(\omega)
$$

Note that in this formulation, assumptions are made about source and receiver characteristics and surface reflectivity that simplify the surface operator to a frequency-dependent scaling factor $A(\omega)$ (see Verschuur et al., 1992; Verschuur and Berkhout, 1997). The advantage of the recursive expression is that the multiple-free upgoing wavefield $\mathbf{P}_{0}^{-}(\omega)$ can be found iteratively, where in each iteration the surface scaling factor $\hat{A}(\omega)^{(n+1)}$ can be estimated. Each iteration thus simplifies to a linear optimization scheme that minimizes the energy in the multiple-free estimate of the data in equation 1 in what is called "adaptive subtraction." When the procedure starts with the measured data $\mathbf{P}^{-}(\omega)$ as the initial estimate of the multiple-prediction operator, the number of iterations needed for a multiple-free result theoretically equals the highest order of surface multiple present in the measured data. In practice, often only one iteration of multiple prediction (and adaptive subtraction) is applied.

\section{D surface-related multiple prediction}

In theory, the matrix formulations for surface-related multiple prediction (SRMP) hold for 2D as well as 3D wavefields. Interpretation of the matrices determines whether $2 \mathrm{D}$ or $3 \mathrm{D}$ wavefields are considered (Kinneging et al., 1989).

Following equation 1 , the calculation of one unscaled multiple trace, i.e., one element $\bar{M}_{i j}^{-}$of the surface-related multiples matrix, defined by

$$
\overline{\mathbf{M}}^{-}(\omega)^{(n)}=\mathbf{P}_{0}^{-}(\omega)^{(n)} \mathbf{P}^{-}(\omega),
$$

involves the inner product of the $i^{\text {th }}$ row of the matrix $\mathbf{P}_{0}^{-}(\omega)^{(n)}$ with the $j^{\text {th }}$ column of the matrix $\mathbf{P}^{-}(\omega)$. If only the summation coordinates at which sources and receivers are present are considered, the prediction of one multiple trace is achieved with

$$
\begin{aligned}
\bar{M} & \left(x_{r}, y_{r}, x_{s}, y_{s}, \omega\right) \\
& =\sum_{y_{k}} \sum_{x_{k}} P_{0}\left(x_{r}, y_{r}, x_{k}, y_{k}, \omega\right)^{(n)} P\left(x_{k}, y_{k}, x_{s}, y_{s}, \omega\right) \\
& =\sum_{y_{k}} \sum_{x_{k}} M_{x y}\left(x_{r}, y_{r}, x_{s}, y_{s}, x_{k}, y_{k}, \omega\right)
\end{aligned}
$$

where $\left(x_{s}, y_{s}\right)$ and $\left(x_{r}, y_{r}\right)$ are the source and receiver coordinates, respectively, and $\left(x_{k}, y_{k}\right)$ are the summation variable coordinates at which a source and a receiver are present. The 3D SRMP is accomplished with a surface-consistent convolution of a 3D common-receiver gather with a 3D commonsource gather, followed by summation of the 3D multiplecontributions.

The two summations of the $3 \mathrm{D}$ multiple contributions can be applied separately. The inline Fresnel summation (over $x_{k}$ ) applied to the 3D multiple-contribution gather (defined as $\left.M_{x y}\right)$ results in the crossline multiple contribution gather.

$M_{y}\left(x_{r}, y_{r}, x_{s}, y_{s}, y_{k}, \omega\right)=\sum_{x_{k}} M_{x y}\left(x_{r}, y_{r}, x_{s}, y_{s}, x_{k}, y_{k}, \omega\right)$,

in which the subscripts denote the variable coordinates over which the gather needs to be summed. The crossline Fresnel summation (over $y_{k}$ ) applied to the crossline multiplecontribution gather results in the (unscaled) $3 \mathrm{D}$ predicted multiple trace

$$
\bar{M}\left(x_{r}, y_{r}, x_{s}, y_{s}, \omega\right)=\sum_{y_{k}} M_{y}\left(x_{r}, y_{r}, x_{s}, y_{s}, y_{k}, \omega\right) .
$$

The multiple prediction is followed by an adaptive subtraction in the space-time domain (Verschuur and Berkhout, 1997).

\section{Practical considerations}

To better understand the multiple prediction process, its requirements for the data, and its limitations, 3D multiple prediction is illustrated using a 3D synthetic-data example.

First, SRMP and SRME are formulated using a forward model for waves that have propagated (downward and upward) and reflected (or refracted or diffracted) in the subsurface. Therefore, all wave phenomena that propagate laterally just below the free surface (direct and surface waves) are 
assumed to have been removed in advance. Dragoset and Jeričević (1998) give a general and extensive overview of the constraints for SRMP and the violations of these constraints in practical situations. In this paper, we concentrate on practical considerations for 3D marine data sets.

For 3D marine data sets, two main factors determine the applicability of 3D SRMP: first, the spatial sampling of sources and receivers along the two spatial coordinates, and second, the areal aperture of the acquired data. The influence of these factors on the multiple-prediction result is illustrated using synthetic data from a 3D model. The model used to generate the data and multiple-prediction operator consists of two dipping plane reflectors (with dips of $-5^{\circ}$ and $10^{\circ}$ in the inline direction for the first and second reflector, respectively, and dips of $5^{\circ}$ and $-5^{\circ}$ in the crossline direction). The velocity of the first layer is $1500 \mathrm{~m} / \mathrm{s}$, and $2000 \mathrm{~m} / \mathrm{s}$ for the second layer.

Calculation of the (densely sampled) multiple contributions for one multiple output trace is accomplished with the surface-consistent convolution in time (i.e., multiplication in frequency) defined in equation 4 . The inline and crossline summation of the 3D multiple contributions $\left(M_{x y}\right)$ described by equations 5 and 6 extracts and translates the 3D multiple information into the actual 3D predicted multiple trace. After the inline summation, the crossline multiple-contribution gather $\left(M_{y}\right)$ can be analyzed on 3D effects of the multiple reflection events. In Figure 1a, the crossline multiplecontribution gather is shown, and the effects of the crossline dipping reflectors on the multiple events are clearly visible: the Fresnel zones (apices of the events) are shifted with respect to the $y_{k}=0 \mathrm{~m}$-axis. In this gather the trace at $y_{k}=0 \mathrm{~m}$ represents the $2 \mathrm{D}$ multiple prediction result.

The 3D predicted multiple trace is obtained after application of the (edge-effect protected) crossline summation (Figure 1c). Correlation of the 3D predicted multiple trace (trace 2) with its corresponding measured data trace (trace 1) confirms the accurate $3 \mathrm{D}$ multiple prediction. This result is obtained using data acquired with the unrealistic geometry of sources and receivers on a $25 \times 25$-m grid with a width of $2000 \mathrm{~m}$ in both spatial directions. In general, the minimum requirements to obtain the accurate $3 \mathrm{D}$ multiple prediction result are the large aperture that covers all the Fresnel zones at the surface (depicted by rectangles in Figure 1a,b) and the alias-protected spatial sampling within the aperture of both sources and receivers.

Because of economic and practical constraints, these minimum data requirements are difficult to realize. Although 3D marine acquisition technology is improving constantly, 3D data acquired today are severely undersampled in the crossline direction for multiple prediction purposes. Typical 3D marineacquisition geometries are designed to have a uniform common-midpoint (CMP) distribution. For that reason, the subsequent sail-lines are typically separated by half the crossline aperture length. As a result of this design, the sampling of sources in the crossline direction can be a few hundred meters. Therefore, straightforward application of 3D SRMP to these 3D marine data sets without severe aliasing effects is impossible.

Because of the sparse crossline sampling of sources, multiple contributions can only be calculated for the few crossline coordinates at which source lines (approximately) coincide with receiver lines. Not only are the crossline multiple contributions sparsely sampled, the crossline aperture is also limited. The crossline Fresnel summation for 3D SRMP cannot be applied because of the introduction of severe aliasing effects combined with the limited aperture edge effects (Fresnel zones outside the measured aperture). To illustrate the aliasing (and edge) effects, a subset of the densely sampled crossline multiple-contribution gather is taken (Figure 1b). The effects of the sparse crossline sampling on 3D SRMP are clearly illustrated in the unacceptable 3D multiple prediction result (trace 3 in Figure 1c).

\section{D MULTIPLE PREDICTION USING SPARSE INVERSION (MPSI)}

\section{Fresnel zone reconstruction}

In principle, for accurate $3 \mathrm{D}$ multiple prediction, only the multiple contributions within the Fresnel zones are required. The effort in calculating densely sampled multiplecontribution gathers is necessary because the lateral locations of the Fresnel zones are unknown: the Fresnel summation will automatically extract them. In Figure 1, the effect of sparse sampling on 3D multiple prediction is demonstrated. Fresnel zone reconstruction, the main topic of this article, aims at extracting the correct multiple information from sparsely distributed crossline multiple contributions.

The starting point of the Fresnel zone reconstruction approach to $3 \mathrm{D}$ multiple prediction is the sparsely sampled crossline multiple-contribution gather (such as shown in Figure $1 \mathrm{~b}$ ), assuming that the inline summation can be applied without introducing severe aliasing artifacts. The multiple information from this gather is extracted using a transformation a)

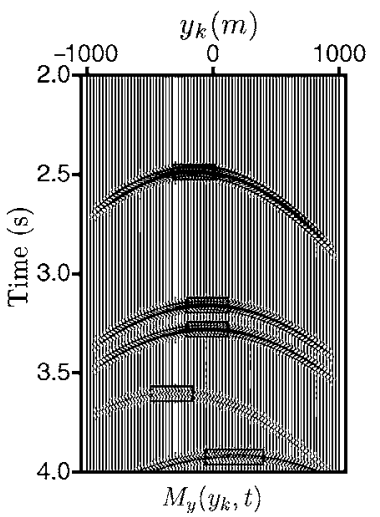

b)

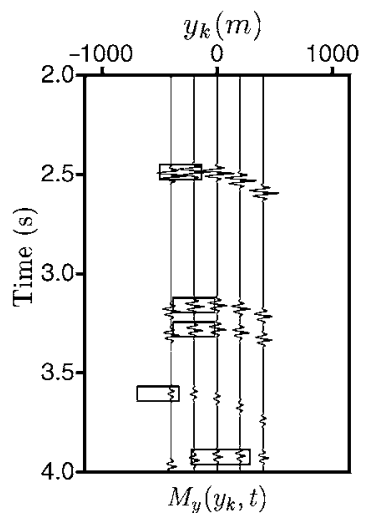

c)

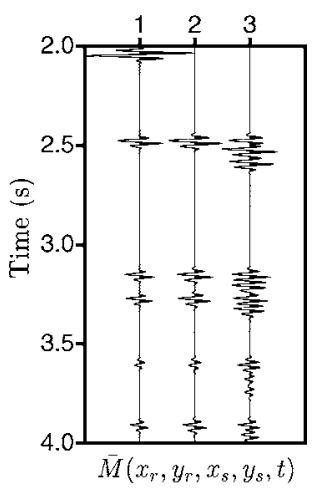

Figure 1. (a) The (edge-tapered) crossline multiple-contribution gather $M_{y}\left(y_{k}, t\right)$ constructed from densely sampled $3 \mathrm{D}$ data. The rectangles indicate Fresnel zones at the surface. (b) The sparsely sampled crossline multiplecontribution gather from (a). (c) The measured data trace 1 with multiples, 3D SRMP result (trace 2) after contribution summation of (a), and the 3D SRMP result (trace 3 ) after contribution summation of (b). 
that maps the crossline multiple-contribution events into a model space containing information necessary for the multiple prediction. This transformation is defined in the next section.

\section{Parameterization of crossline multiple contributions}

Replacing the crossline summation $\left(\sum_{y_{k}}\right)$ in equation 6 with a transformation requires an appropriate parameterization of the (crossline) multiple contributions. The goal is to extract the multiple information from available multiple contributions using this transformation. The information that should be extracted consists of the apex traveltimes (traveltimes of the stationary multiple contributions), the apex positions (lateral locations of the apices of the multiple-contribution events), and the curvatures (amplitudes and curvatures of the multiple-contribution events).

A possible choice for the curvature parameter is to assume that the multiple contributions have a hyperbolic moveout (see, e.g., Figure 1a). In Appendix A, the hyperbolic moveout assumption for crossline multiple contribution events is verified for a few simple cases. The hyperbolic assumption is exact for the crossline multiple contributions of the first-order multiple from a horizontal reflector, from a source-receiver pair at the same crossline coordinate. In general, the moveout of the crossline multiple-contribution events will deviate from the hyperbolic traveltime expression (e.g., in the case of a crossline offset between source and receiver for different types of multiples, or if the subsurface has crossline structure). Note, however, that we only need to describe the Fresnel zone of the crossline multiple-contribution events around the apex. The apex itself can be located at any crossline location. The numerical experiments in Appendix A show that the deviations are moderate and that the hyperbolic moveout assumption can still be used to parameterize the crossline multiplecontribution events.

The crossline multiple contributions are thus parameterized with three parameters: the traveltime of the stationary multiple contribution (i.e., the intercept time $\tau$ ), the crossline a)

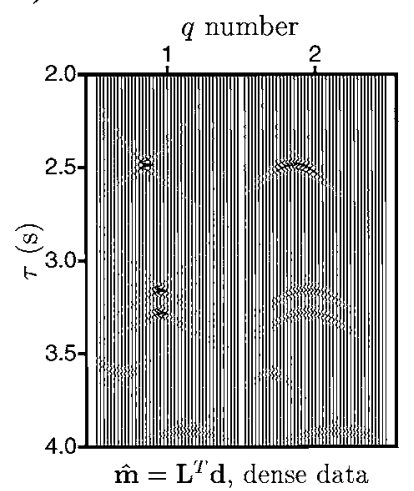

b)

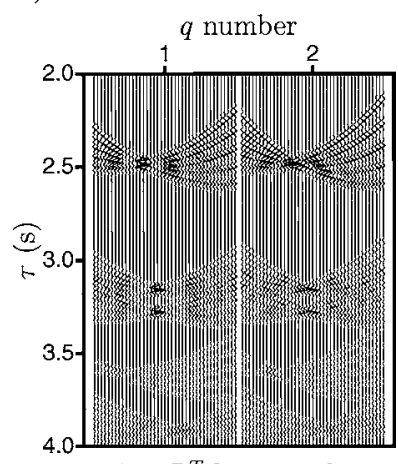

$\hat{\mathbf{m}}=\mathbf{L}^{T} \mathbf{d}$, sparse data
Figure 2. (a) Estimated model space $\hat{m}\left(q, y_{0}, \tau\right)$ obtained after applying the adjoint transformation $\left(\mathbf{L}^{T}\right)$ to the (limited) input data from Figure 1a $\left(2 q\right.$-values, $y_{0}=-500$ to $\left.+500 \mathrm{~m}\right)$. (b) Estimated model space $\hat{m}\left(q, y_{0}, \tau\right)$ obtained after applying the adjoint transformation to the (sparse) input data from Figure $1 \mathrm{~b}$ (two $q$-values, $y_{0}=-500$ to $+500 \mathrm{~m}$ ). apex position of the multiple contributions $y_{0}$, and the hyperbolic curvature of the crossline multiple-contribution traveltime function $q\left(=1 /(v / 2)^{2}\right)$ around the apex. With this parameterization, the transformations from data space (crossline multiple contributions) to model space $\left(q, y_{0}, \tau\right)$ can be defined.

The transformation from the data space $d\left(y_{k}, t\right)$, representing the crossline multiple-contribution gather as a function of crossline position and time, to the model space $m\left(q, y_{0}, \tau\right)$, containing the multiple information present in the crossline contribution gather, is achieved with the following linear operation:

$$
\hat{m}\left(q, y_{0}, \tau\right)=\sum_{y_{k}=y_{k, \min }}^{y_{k}=y_{k, \max }} d\left(y_{k}, t=\sqrt{\tau^{2}+q\left(y_{0}-y_{k}\right)^{2}}\right),
$$

which can be written in operator notation as

$$
\hat{\mathbf{m}}=\mathbf{L}^{T} \mathbf{d}
$$

where ${ }^{T}$ is the adjoint. Similarly, the data can be modeled using the transformation from model to data space, which is achieved with the following operation:

$$
\begin{aligned}
& d\left(y_{k}, t\right) \\
& =\sum_{q=q_{\min }}^{q=q_{\max }} \sum_{y_{0}=y_{0, \min }}^{y_{0}=y_{0, \max }} m\left(q, y_{0}, \tau=\sqrt{t^{2}-q\left(y_{0}-y_{k}\right)^{2}}\right),
\end{aligned}
$$

or in operator notation

$$
\mathbf{d}=\mathbf{L m}
$$

The size of the transformation operator $\mathbf{L}$ is the number of data points $\left(N_{d}=N_{y_{k}} \times N_{t}\right)$ times the number of model parameters $\left(N_{m}=N_{q} \times N_{y_{0}} \times N_{\tau}\right)$.

Ideally, the adjoint transformation $\left(\mathbf{L}^{T}\right)$ maps all hyperbolic trajectories in the crossline multiple-contribution gather (d) into points in the model space ( $\hat{\mathbf{m}})$. However, because of the finite aperture and discretization of the crossline multiplecontribution gathers, these points in the model space are smeared. The smearing caused by the finite aperture of the wavefield is illustrated on the densely sampled crossline multiple-contribution gather from Figure 1a. The smearing in the 3D model space (with two $q$-values) introduced by the transformation is clearly visible in Figure 2a.

Moreover, the crossline multiple-contribution gathers are not expected to be densely sampled over a large crossline offset range. More likely, in practice only a few of the crossline contributions are available as input for the transformation (Figure 1b). The sparse sampling of the input data introduces severe aliasing and smearing artifacts in the model space, thereby further degrading the estimated model space. The effects of sparse sampling combined with limited aperture of the input data on the transformation to the model space are illustrated in Figure 2b. The resulting model space is far from the desired result, in which the crossline multiplecontribution events in the input data are mapped into isolated wavelets in the model space, corresponding to the stationary contributions.

A very similar problem is observed when estimating the high-resolution velocity space from CMP gathers with a 
finite aperture (Thorson and Claerbout, 1985; Sacchi and Ulrych, 1995). The transformations of equations 8 and 10 follow the transformations defined in the problem of estimating the high-resolution velocity gather, with the difference that in the problem at hand, the hyperbolic events have an additional degree of freedom in the lateral location of the apex (which results in an additional dimension in $\mathbf{m}$, defined as $y_{0}$ ). The solution to the problem of smearing in the velocity space is found by defining a better estimate to the inverse of the modeling operator $\mathbf{L}$ than the low-resolution adjoint operator $\mathbf{L}^{T}$. For this purpose, the transformation from data to model space is redefined as an inverse problem.

The relation between data space and model space in the presence of noise is given by

$$
\mathbf{d}=\mathbf{L m}+\mathbf{n},
$$

where $\mathbf{n}$ represents the noise term present in the data, which is assumed to be random noise with a Gaussian probability density function (pdf). A solution to this naturally ill-posed problem is found by regularizing the inverse problem by incorporating a priori information. The desired solution is the highresolution solution, containing a sparse set of wavelets and representing the apices in the crossline multiple-contribution gather. By imposing a Cauchy pdf on the model space parameters, the goal, i.e., sparseness in the model space, isbreak achieved.

\section{Cauchy probability density function}

The use of the Cauchy pdf imposed on the model space parameters not only regularizes the inversion but also enhances the resolution in the model space. The more popular Gaussian regularizer, on the other hand, tends to smear out the energy in the model space (Sacchi et al., 1998; Zwartjes and Duijndam, 2000). By imposing a Cauchy pdf on the model space parameters, the majority of the model space parameters must take a value close to zero, whereas the outliers make up the significant model space parameters.

The induced sparseness property of the Cauchy pdf is often used in the regularization of inverse problems in which a highresolution model space is the objective. Sacchi et al. (1998) use the Cauchy criterion to address smearing in the frequency domain caused by the finite aperture of the measurements. The resolution in the model space is increased by imposing the Cauchy norm on the model space parameters. Similar results are obtained by Zwartjes and Duijndam (2000), who use the Cauchy norm as a regularizer in reconstruction of sparsely and irregularly sampled seismic data.

\section{Sparse inversion of crossline multiple contributions}

Incorporation of the Cauchy norm in the least-squares inverse problem stated in equation 11 yields the maximum a posteriori (MAP) estimator $\hat{\mathbf{m}}$ :

$$
\hat{\mathbf{m}}=\left(\mathbf{L}^{T} \mathbf{L}+\lambda \mathbf{Q}^{-1}\right)^{-1} \mathbf{L}^{T} \mathbf{d},
$$

where $\lambda=\sigma_{n}^{2} / \sigma_{m}^{2}$, and $\mathbf{Q}$ is an $N_{m} \times N_{m}$ diagonal matrix with elements

$$
Q_{i i}=1+\frac{m_{i}^{2}}{\sigma_{m}^{2}}, \quad i=1, \ldots, N_{m}
$$

Equation 12 resembles the damped least-squares solution to the inverse problem in equation 11 , with the difference that the damping term $\lambda \mathbf{Q}^{-1}$ is a function of the solution $\mathbf{m}$. Incorporation of a priori information turns the linear inverse problem into a nonlinear one that requires a nonlinear optimization method to be solved.

The solution $\mathbf{m}$ to the nonlinear inversion is controlled by two important parameters: $\sigma_{n}$ and $\sigma_{m}$. The first parameter $\sigma_{n}$ is a measure for the (Gaussian) noise present in the data. This measure contains random noise in the data as well as noise caused by modeling uncertainties and measurement errors assumed to be uncorrelated. The second parameter $\sigma_{m}$ controls the degree of sparseness in the solution. When the ratio $m_{i}^{2} / \sigma_{m}^{2}$ is large, the stabilization or damping imposed on the model parameter $m_{i}$ is small. On the other hand, when the ratio is small, the model parameter will be damped, i.e., will approach zero value. The mechanism enhances only the model parameters that are relevant in fitting the data and, therefore, increasing the resolution of the model space. The two parameters are linked through the parameter $\lambda$, which controls the stabilization (or the trade-off between sparseness and data fitting) in the inversion. In practice, the parameter $\sigma_{n}$ is kept fixed, and the parameter $\sigma_{m}$ is used to fine-tune the inversion.

The nonlinear system of equations, shown in vector notation in equation 12, is solved using a nonlinear conjugate gradient (CG) optimization method (Shewchuk, 1994).

\section{From model space to multiple trace}

Replacing the crossline Fresnel summation of multiple contributions in equation 6 with a nonlinear parametric inversion requires additional operations to translate the $3 \mathrm{D}$ multiple information in the resulting model space into the actual 3D predicted multiple trace. A straightforward method to translate the multiple information in the model space into the multiple trace is to model densely sampled crossline multiple contributions using the modeling operator $(\hat{\mathbf{d}}=\mathbf{L} \hat{\mathbf{m}})$, after which the crossline summation can be applied. However, this result can be directly obtained from the model space information by using the stationary phase approximation.

The stationary phase approximation (Bleistein, 1984; Wapenaar, 1992) used for the purpose of multiple prediction is achieved through an amplitude and phase correction applied to the model space. The correction is a function of the model space parameters $q$ and $\tau$ (and temporal frequency) and is given by

$$
F_{\text {corr }}(q, \tau)=\sqrt{\frac{2 \pi \tau}{q \omega}} e^{-j(\pi / 4)} .
$$

The resultant summation of the model space traces after the correction projects the corrected stationary crossline multiple contributions in the model space directly onto the multiple trace $\bar{M}_{\text {MPSI }}\left(x_{r}, y_{r}, x_{s}, y_{s}, t\right)$ :

$\bar{M}_{\mathrm{MPSI}}\left(x_{r}, y_{r}, x_{s}, y_{s}, t\right)=\sum_{q_{\min }}^{q_{\max }} \sum_{y_{0, \min }}^{y_{0, \max }} F_{c o r r}(q, \tau) \hat{\mathbf{m}}\left(q, y_{0}, \tau\right)$.

This step concludes the 3D MPSI procedure, in which the crossline Fresnel summation of multiple contributions is 
replaced with a parametric inversion followed by an amplitude and phase correction and summation in the model domain.

\section{Illustration of 3D multiple prediction using sparse inversion (MPSI)}

To illustrate the 3D MPSI method, we start with the sparsely sampled crossline multiple-contribution gather from Figure 1b. The model space is defined with two $q$-values (based on the analysis in Appendix A), an apex coordinate range from $y_{0}=-500$ to $+500 \mathrm{~m}$ sampled at every $25 \mathrm{~m}$, and an intercept time range and sampling similar to the sampling of the input data.

In Figure 3a-c, snapshots of the 3D model space during the sparse inversion iteration process are displayed, illustrating how the sparseness measure reduces smearing. The final estimate of the 3D model space contains approximately five isolated wavelets, corresponding to the stationary contributions in the densely sampled crossline multiple-contribution gather (in Figure 1a). The 3D MPSI trace is shown as trace 1 in Figure $3 \mathrm{~d}$. Compared to the full 3D SRMP result (trace 2), the differences between the two results, being mainly amplitudes, are very small (trace 3 ). Amplitude differences are credited to the fact that different contribution events cannot be described perfectly with the two curvature parameters. The amplitude correction using the $q$-values from the inversion is not valid for all the events; therefore, small amplitude differences occur.
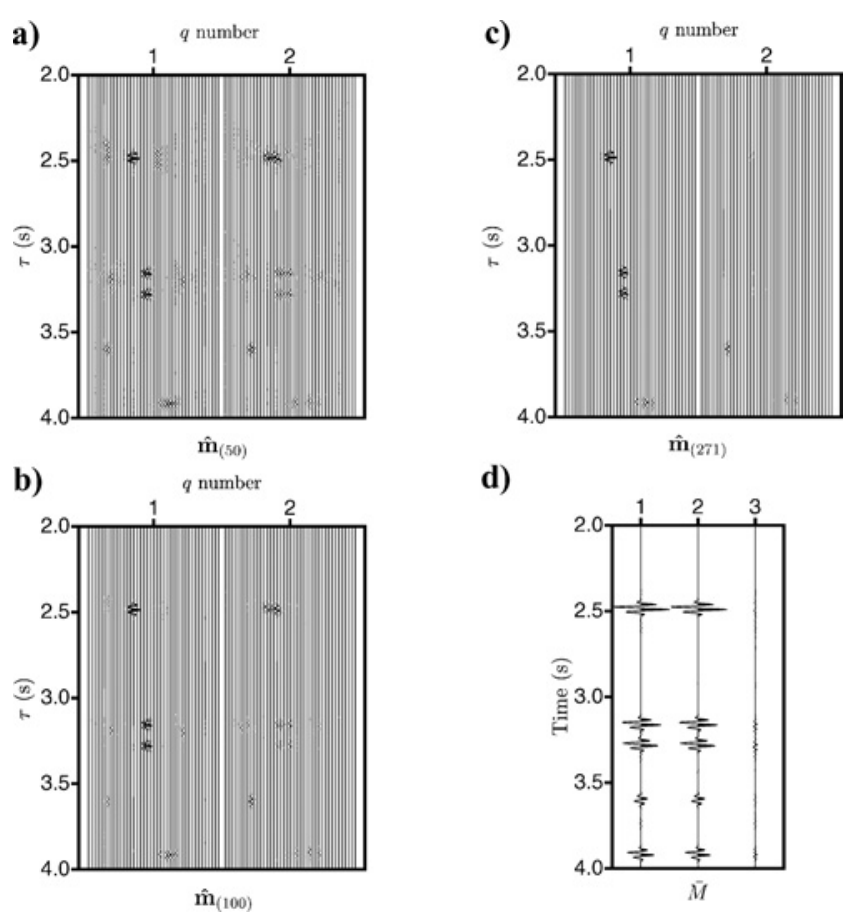

d)

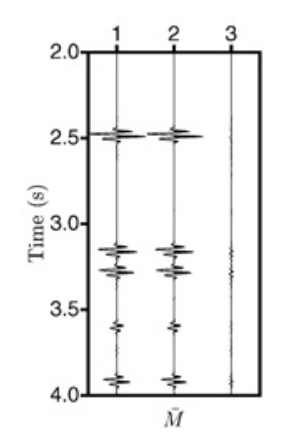

Figure 3. Verification of the MPSI methodology on a crossline multiple-contribution gather from the data of Figure $1 \mathrm{~b}$. (a) Snapshot of the 3D model space after 50 iterations $\hat{\mathbf{m}}_{(50)}\left(q, y_{0}, \tau\right)$. (b) Snapshot of the 3D model space after 100 iterations $\hat{\mathbf{m}}_{(100)}\left(q, y_{0}, \tau\right)$. (c) Final estimate of the 3D model space $\hat{\mathbf{m}}\left(q, y_{0}, \tau\right)$ after 271 iterations. (d) 3D MPSI result (trace 1), 3D SRMP result (trace 2 ), and difference (trace 3 ).
More important are the correct traveltimes that are resolved in the sparse inversion.

In the next sections, the 3D MPSI method is demonstrated on a synthetic 3D data set and a 3D marine data set from offshore Norway.

\section{D SYNTHETIC-DATA EXAMPLE}

\subsection{D model and 3D surface seismic data}

The 3D synthetic data are generated on a model that varies only in the crossline direction $y$ and is invariant in the inline direction $x$. The model is based on a typical Gulf of Mexico environment: deep water with a relatively shallow salt structure. A cross section of the model impedance $\rho v$ is shown in Figure 4. The main characteristics of the model are the relatively flat, strongly reflective sea bottom, the upside-down-foot-shaped salt structure with very steep flanks and large impedance contrast, and a number of weak reflectors resembling the sediments. Note that discontinuities in the definition of the sea bottom and salt structure act as diffractive structures because of the gridding of the model.

Densely sampled 3D surface seismic data are generated for the $2.5 \mathrm{D}$ model using a 3D finite-difference acoustic modeling code (the same modeling code used in Etgen and Regone, 1998). Each 3D shot record contains 81 streamers separated by $50 \mathrm{~m}$ in the crossline direction, and with receivers sampled at every $35 \mathrm{~m}$ in the inline direction. Since the model is invariant in the inline direction, repeated $3 \mathrm{D}$ shot records simulate sail-lines. In total, 240 sail-lines, separated by $100 \mathrm{~m}$ in the crossline direction, are modeled using this geometry. The acquisition starts at $y=7000 \mathrm{~m}$ and ends at $y=30900 \mathrm{~m}$, which results in incomplete $3 \mathrm{D}$ shot records at the edges of the model.

For the experiment, the multiples for the zero-offset section from $y_{r}=7000 \mathrm{~m}$ to $y_{r}=30900 \mathrm{~m}$ (at every $100 \mathrm{~m}$ ) are predicted in a true 3D sense, using different subsets of the 3D data set. The zero-offset section is shown in Figure 5. Since the acquisition geometry and the sail direction are oriented perpendicular to the zero-offset section, the $3 \mathrm{D}$ effects in the zero-offset section originate only from the crossline structure of the model.

The zero-offset section is dominated by seismic events from two highly reflective (and diffractive) interfaces: the sea bottom and the salt structure. Strong out-of-plane diffraction events originate from the gridding of the ocean bottom and

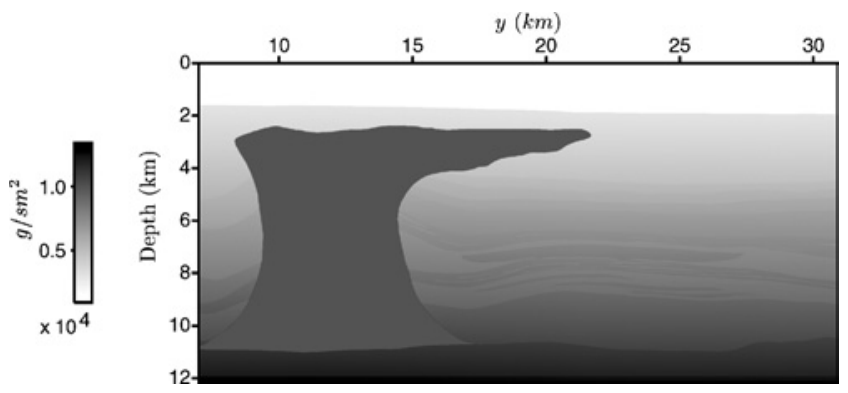

Figure 4. The 2.5D Gulf of Mexico model on which 3D data are acquired (courtesy BP Upstream Technology Group). Shown is a cross section at the constant $x$-coordinate of the model impedance $\rho v$. 
salt structure in the model. Both interfaces generate a train of surface (and internal) multiples. The primary reflections (and diffractions) from the sediments are obscured by the train of (out-of-plane) multiples from the sea bottom and salt structure, reflecting the necessity of an accurate $3 \mathrm{D}$ prediction of these multiples. Some of the weak sediment reflections are observed just below the first-order sea-bottom reverberation at $y_{r}=26000 \mathrm{~m}$.

In the next section, the available crossline multiple contributions for the multiples in the zero-offset section, are calculated, and the 3D effects of the multiples are analyzed. The limitations of the 2D SRMP approach for this 3D data set are illustrated.

\section{Crossline multiple contributions}

The 3D multiple contributions $\left(M_{x y}\right)$ for the zero-offset multiple-output traces are sampled at every $35 \mathrm{~m}$ in the inline direction and at every $100 \mathrm{~m}$ in the crossline direction. The crossline multiple contributions $\left(M_{y}\right)$ are obtained after the inline Fresnel summation of the 3D multiple contributions. Note that the temporal frequency of the signal does not exceed 20 Hz. Depending on the crossline coordinate of the multipleoutput trace (with respect to the boundaries of the survey), the crossline multiple-contribution gather contains 21 to 41 contributions with a crossline aperture of, at most, $4000 \mathrm{~m}$.

In Figure 6, the available crossline multiple contributions are shown for the multiple-output traces at $y_{r}=8000,16000$

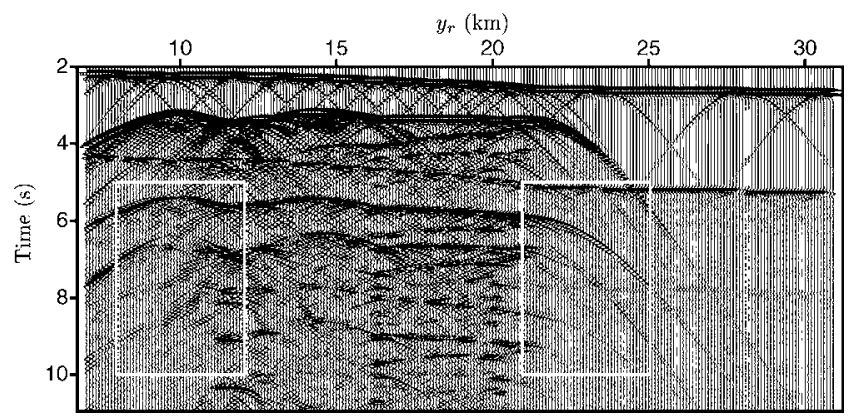

Figure 5. The zero-offset section for which the multiples are predicted. a)

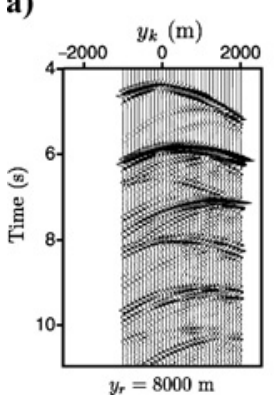

b)

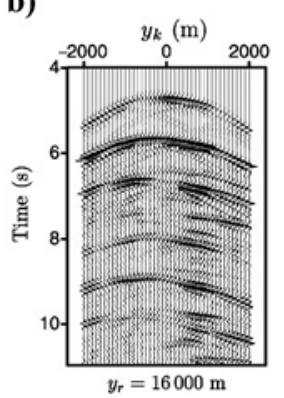

c)

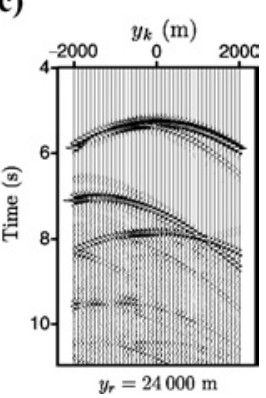

Figure 6. Densely sampled crossline multiple-contribution gathers for three zero-offset multiple output traces. (a) Crossline multiple contributions for the multiple trace at $y_{r}$ $=8000 \mathrm{~m}$, (b) $y_{r}=16000 \mathrm{~m}$ and (c) $y_{r}=24000 \mathrm{~m}$. Note that the trace at $y_{k}=0 \mathrm{~m}$ in each panel represents the 2D SRMP result for the zero-offset data trace. and $24000 \mathrm{~m}$. The zero offset traces are chosen at three typical model locations: to the left of the salt structure, on top of the salt structure, and to the right of the salt structure. The $3 \mathrm{D}$ character of the surface multiples can be analyzed in the (densely sampled) crossline multiple-contribution gathers, as the apex of each event in the crossline multiple-contribution gather represents the crossline reflection position at the surface of that particular multiple event. Note that even with this unrealistic dense acquisition, full 3D SRMP, achieved by summing all traces in each panel of Figure 6, results in some aliasing artifacts.

The 2D SRMP result is identical to the crossline multiple contribution at $y_{k}=0 \mathrm{~m}$, for which traveltime and amplitude errors are obvious. The traveltime and amplitude errors in the 2D prediction are clearly illustrated in two close-ups (white rectangles in Figure 5) in Figures $7 \mathrm{~b}$ and $8 \mathrm{~b}$. The surface multiples generated at the relatively horizontal reflectors have been predicted at the correct traveltimes; large traveltime errors occur in multiples from diffraction events and at the edges of the salt. Note that amplitude differences in the predicted higher-order multiple events may also be the result of carrying out only the first iteration of the iterative SRMP approach (Berkhout and Verschuur, 1997).

The 2D surface-related multiple elimination (SRME) result for the zero-offset section is shown in Figures $7 \mathrm{f}$ and $8 \mathrm{f}$. Only the sea-bottom reverberations and the multiple reflections from the relative horizontal part of the salt structure are well suppressed; the out-of-plane multiples are barely suppressed (as pointed out by the arrows in Figures 7 and 8).

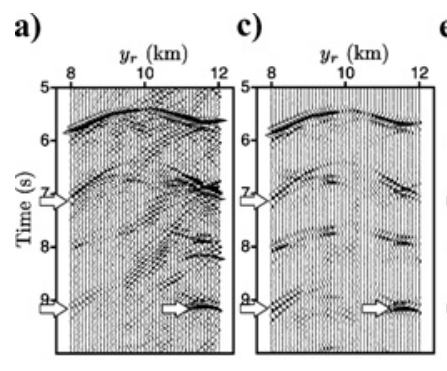

b)

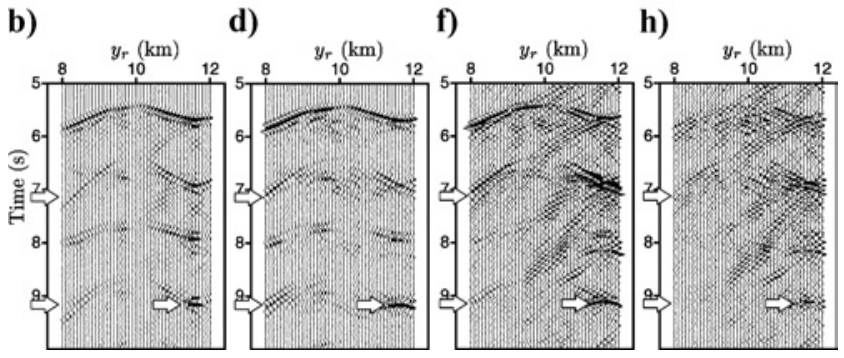

d)

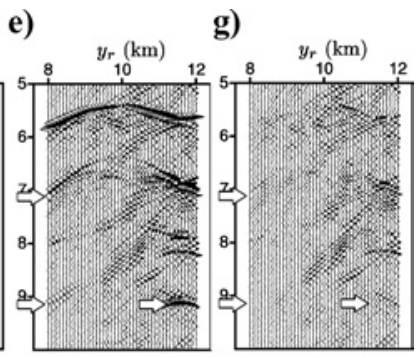

Figure 7. Comparison of the 2D SRMP and 3D MPSI results, zoomed in on the left of the salt structure in the zero-offset section (see Figure 5 for the zoom area). The arrows point to multiples that are well predicted by the $3 \mathrm{D}$ method and wrongly predicted by the 2D method. (a) The zero offset section. (b) The 2D SRMP result. (c) The 3D MPSI result, obtained with (at most) 21 contributions. (d) The 3D MPSI result obtained with (at most) 9 contributions. (e) The zero offset section. (f) The 2D SRME result. (g) The 3D MESI result, obtained with (at most) 21 contributions. (h) The 3D MESI result, obtained with (at most) 9 contributions. 


\section{D MPSI for the zero-offset section}

The 3D MPSI method is illustrated on the zero-offset section using two different subsets of the available crossline multiple contributions. First, spatial sampling of the crossline multiple-contribution gathers is increased to $200 \mathrm{~m}$, to verify the 3D MPSI procedure. Then, 3D MPSI is applied to crossline multiple-contribution gathers with a reduced crossline aperture, sampled at every $400 \mathrm{~m}$.

\section{D MPSI using 21 crossline multiple contributions}

The 3D MPSI procedure is first illustrated using relatively densely sampled crossline multiple-contribution gathers as input for the sparse inversion. However, the aperture of the subset is too small and the sampling is too coarse for full 3D SRMP.

The subset of the crossline contributions for the multipleoutput trace at $y_{r}=8000 \mathrm{~m}$ is shown in Figure 9a. At least two distinct hyperbolic moveouts of the contribution events can be observed: the moveout of the contribution events from (1) reflectors and from (2) diffractive structures. The hyperbolic moveout curvature of the reflection events has a value related to approximately half the water velocity $[\sim q=1.8 \times$ $\left.10^{-6}\left(\mathrm{~s}^{2} / \mathrm{m}^{2}\right)\right]$; for the contribution events from the diffractive structures, the curvature parameter is chosen to be $q=2.8 \times$ $10^{-6}\left(\mathrm{~s}^{2} / \mathrm{m}^{2}\right)$. These two curvature parameters are used to define the 3D model space to be scanned.

As observed in the crossline multiple-contribution gathers for multiple-output traces at different coordinates (Figure 6), at the edges of the salt structure, the apices of the contribution events are shifted toward the salt structure. Therefore,

a)

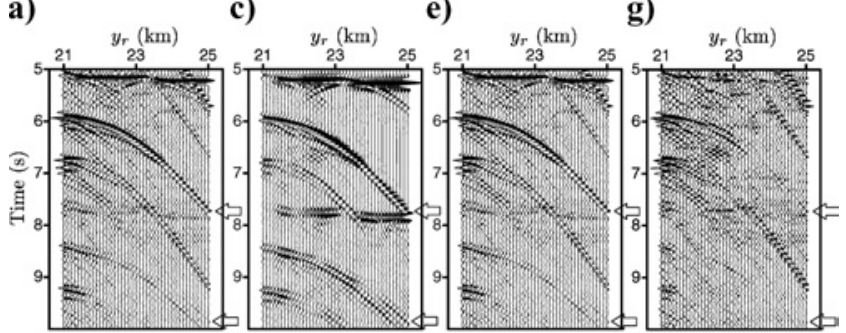

b)

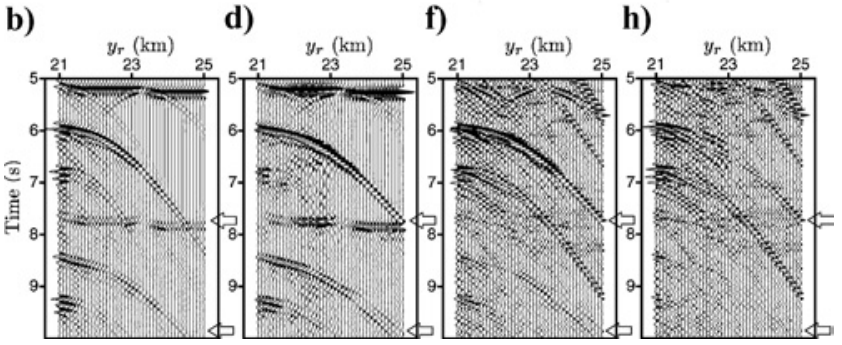

Figure 8. Comparison of the 2D SRMP and 3D MPSI results, zoomed in on the right of the salt structure in the zero-offset section (see Figure 5 for the zoom area). The arrows point to multiples that are well predicted by the $3 \mathrm{D}$ method and wrongly predicted by the 2D method. (a) The zero-offset section. (b) The 2D SRMP result. (c) The 3D MPSI result, obtained with (at most) 21 contributions. (d) The 3D MPSI result obtained with (at most) 9 contributions. (e) The zero-offset section. (f) The 2D SRME result. (g) The 3D MESI result, obtained with (at most) 21 contributions. (h) The 3D MESI result, obtained with (at most) 9 contributions. the model space range of apex coordinates is shifted accordingly. The sampling of apex coordinates is fixed at $50 \mathrm{~m}$. The apex time range is equal to the crossline contribution traveltime range.

The 3D MPSI procedure is illustrated for the multipleoutput trace at $y_{r}=8000 \mathrm{~m}$ in Figures $9 \mathrm{a}-\mathrm{c}$. The estimated model space for this multiple-output trace contains isolated wavelets at both $q$-values, most of which are shifted toward the higher crossline coordinates, i.e., toward the edge of the salt. Note that amplitude variations along contribution events (which have not been included explicitly in the forward model) are described with multiple model space entries. The 3D MPSI result is obtained after applying the appropriate amplitude and phase corrections and summing these corrected model space traces; it is shown as trace 2 in Figure 9c. The 3D MPSI result is compared to the zero-offset data trace (trace 1) and the $2 \mathrm{D}$ SRMP result (trace 3 ). In contrast to the $2 \mathrm{D}$ result, the traveltimes of the $3 \mathrm{D}$ predicted multiple events correspond very well with the measured multiple events in the zero-offset data trace (e.g., note the multiple events at $t \approx 7.0$, 9.1, and $10.0 \mathrm{~s})$.

The 3D MPSI and 2D SRMP results for the two close-up areas (indicated by the white frames in Figures 5) are shown in Figures 7 and 8. The 3D predicted multiples for the close-up area on the left of the salt structure (Figure 7) follow closely the multiples in the zero-offset data, both in amplitude and traveltime. The 2D SRMP result has wrongly predicted the a)

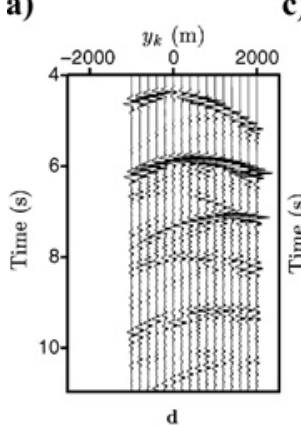

b)

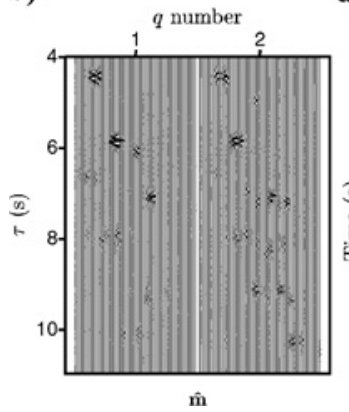

c)

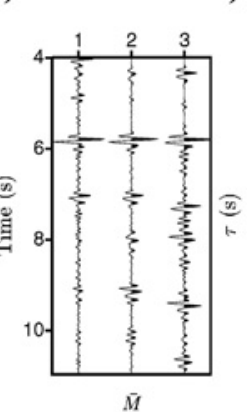

d)

e)

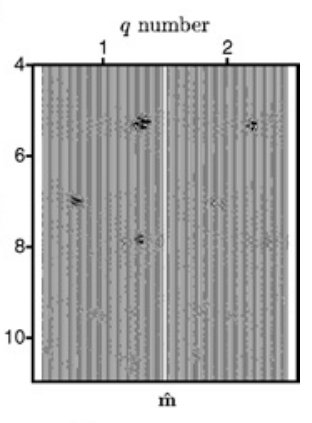

f)

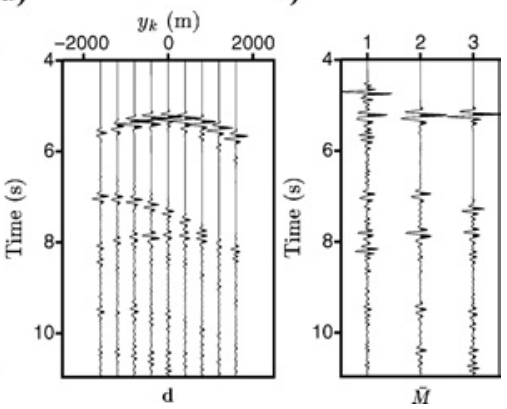

Figure 9. Illustration of 3D MPSI of the multiple-output trace at $y_{r}=8000 \mathrm{~m}$ using 16 contributions $(\mathrm{a}-\mathrm{c})$ and at $y_{r}=24$ $000 \mathrm{~m}$ using 9 contributions (d-f). (a) The 16 crossline multiple contributions used as input for the inversion. (b) The final estimate of the 3D model space (the $q$-values are numbered). (c) The zero-offset data trace 1,3D MPSI result 2, and 2D SRMP result 3. (d) The 9 cross-line multiple contributions used as input for the inversion. (e) The final estimate of the 3D model space (the $q$-values are numbered). (f) The zero-offset data (trace 1), 3D MPSI result (trace 2), and 2D SRMP result (trace 3). 
traveltime of the out-of-plane multiples far from the edge of the salt. Examples of $2 \mathrm{D}$ wrongly predicted multiples are the first edge of the salt multiple $(t \approx 7.3 \mathrm{~s})$ and its split peg-leg multiples $(\mathrm{t} \approx 9.2$ and $9.4 \mathrm{~s})$ at $y_{r}=8000 \mathrm{~m}$. The synclinal structure on the top of the salt at $y_{r} \approx 11600 \mathrm{~m}$ focuses and defocuses multiple energy, which can be observed at $t \approx 6.9,7.8$, and $9.0 \mathrm{~s}$. The focusing and defocusing, which are inherently 3D effects, are correctly predicted in the 3D MPSI result (see the arrows in Figure 7c), as opposed to the 2D SRMP result. Note that all above-mentioned events are barely suppressed in the 2D SRME result (Figure 7f).

In the close-up at the right of the salt structure (Figure 8), interesting 3D features are multiples from the pinch-out of the salt structure and 3D diffracted multiple events from the sea bottom. The 2D SRMP result is fairly correct on top of the salt and deteriorates farther away from the salt pinch out: the tails of the multiple events related to the salt are not correctly predicted in time. Also, the $2 \mathrm{D}$ prediction of the diffraction multiples deteriorates away from the apex of the diffraction multiples. The 3D MPSI result has correctly predicted most of the out-of-plane multiple events in time (as pointed out by the arrows), but the amplitudes are not very accurate. The amplitudes of the first-order salt peg-leg multiple and the first (top and bottom) salt multiple are not correct near the pinch out. Despite the mismatches between the 3D MPSI result and the zero-offset data, the 3D MESI (multiple elimination using sparse inversion) result in Figure $7 \mathrm{~g}$ is better than the 2D SRME result.

The 3D MPSI results in this section were obtained using, at most, 21 crossline multiple contributions. In the next section, the crossline sampling of multiple contributions is increased, and the crossline aperture is reduced.

\section{Illustration of $3 D$ MPSI using nine crossline multiple contributions}

In this section, 3D MPSI is illustrated using, at most, nine crossline multiple contributions, sampled at every $400 \mathrm{~m}$ with an aperture range that extends from (at most) -1600 to $+1600 \mathrm{~m}$.

The 3D model space to be scanned is equal to the model used in the previous section. Figure $9 \mathrm{~d}-\mathrm{f}$ shows the sparse inversion result for the trace at $y_{r}=24000 \mathrm{~m}$. Despite the coarser sampling, a good estimate of the model space is obtained, and the resulting 3D predicted multiple trace matches the input much better than the $2 \mathrm{D}$ result. In the 3D MPSI result for the two windows in the zero-offset section (Figures $7 \mathrm{~d}$ and $8 \mathrm{~d}$ ), the main surface multiple events, i.e., the water-bottom reverberations and the top (and bottom) of the salt multiples, are predicted. The failure to predict the lowamplitude and highly scattered multiple events is a result of the limited crossline multiple information available for the inversion combined with the sparseness imposed on the model parameters. The surface multiples that are not (accurately) predicted include: water-bottom diffraction multiples, multiples from the bottom of the salt, and focused and defocused multiple events (at $y_{r} \approx 11600 \mathrm{~m}$ ).

In Figure 7d, the 3D MPSI result in the close-up left of the salt structure is shown. The out-of-plane multiples at the edge of the salt are accurately predicted. Even the focusing and defocusing effects are reasonably resolved. The multiples are, therefore, better suppressed in the 3D MESI result shown in Figure $7 \mathrm{~h}$ than in the 2D SRME result.

In the close-up to the right of the salt structure, the 3D MPSI procedure has accurately predicted the multiples in time, but amplitude variations along the events are observed (Figure 8d). These variations may be caused by the independent prediction of each multiple output trace. The predicted amplitudes of the sea-bottom multiples are not in relation with the other multiple events. After the adaptive subtraction, remnant multiple energy related to the sea bottom is observed in Figure $8 \mathrm{~h}$. Still, the 3D MESI result is superior compared to the 2D SRME result.

\section{D MARINE DATA EXAMPLE}

The MPSI methodology is tested on a 3D marine data set from an offshore area in mid-Norway. The 3D dual-source survey is acquired with a conventional acquisition geometry designed with a uniform distribution of midpoints. The two sources are fired alternately (flip-flop) at every $25 \mathrm{~m}$ in the sail direction and are separated by $50 \mathrm{~m}$ in the crossline direction. The geometry contains 10 streamers separated by $100 \mathrm{~m}$, with an inline receiver spacing of $12.5 \mathrm{~m}$. Subsequent sail-lines are separated by $500 \mathrm{~m}$.

\section{Preprocessing}

As a first preprocessing step, the inline source interval per source line (either flip or flop) is reduced from 50 to $25 \mathrm{~m}$ with an interpolation method based on automatic event tracking that uses the lower temporal frequencies (described in Appendix B of Anderson, 1996) applied on common-offset sections. After the inline infill of sources, the (inline) nearoffset data traces are restored using the parabolic Radon near-offset restoration technique, described in Kabir and Verschuur (1995). The restoration is applied per sourceline and per streamer in the (inline) common-midpoint-offset domain after an approximate NMO correction.

In this paper, the 3D MPSI method is illustrated for one common-offset section of an outer streamer (shown in Figure 10). For this streamer, only one source line is selected, and

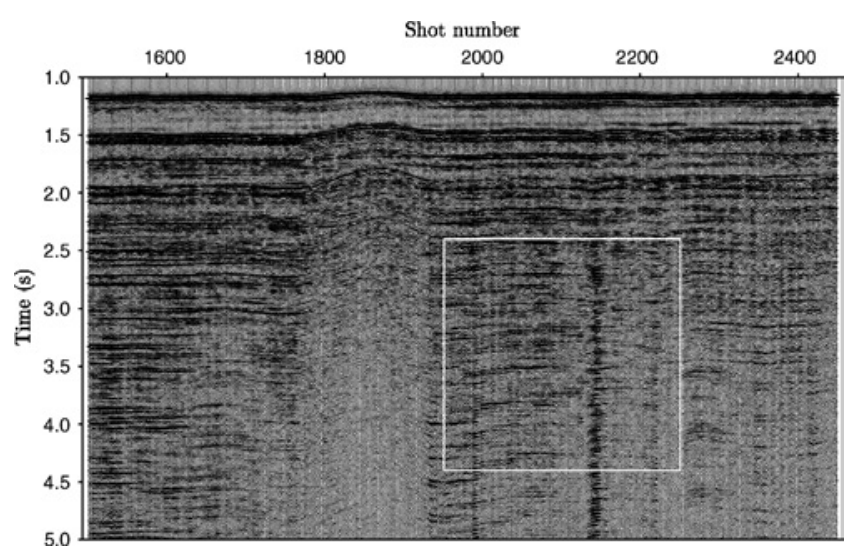

Figure 10. The input data common-offset section measured on the streamer for which the multiples are predicted ( $t^{2}$ gain applied for display). 
a)

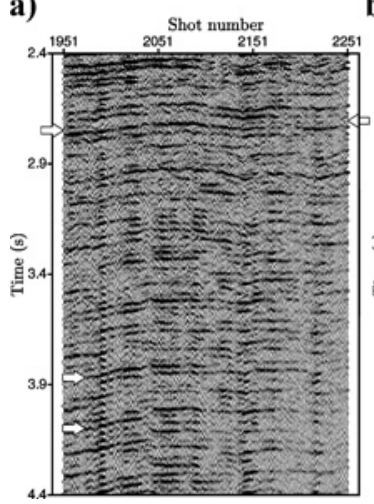

b)

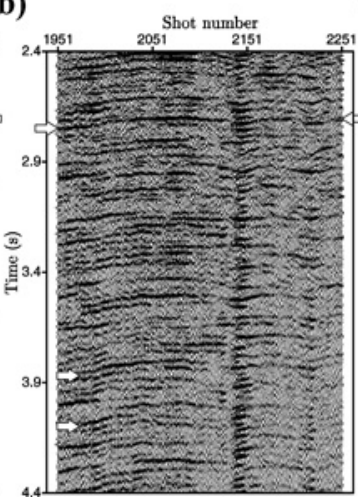

c)

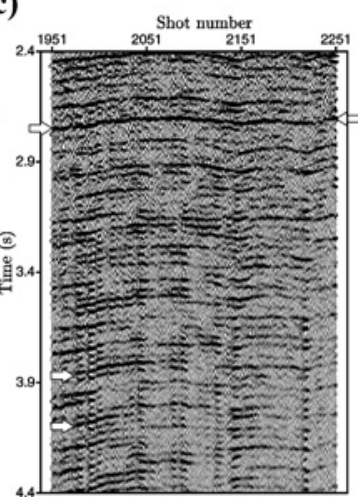

Figure 11. (a) Comparison of the 2D SRMP result, (b) the input data commonoffset section, and (c) the 3D MPSI result, zoomed in on the right of the input data common-offset section (see Figure 10 for the zoom area). The arrows point to multiple reflections that are better predicted by the 3D method than by the $2 \mathrm{D}$ method.

a)

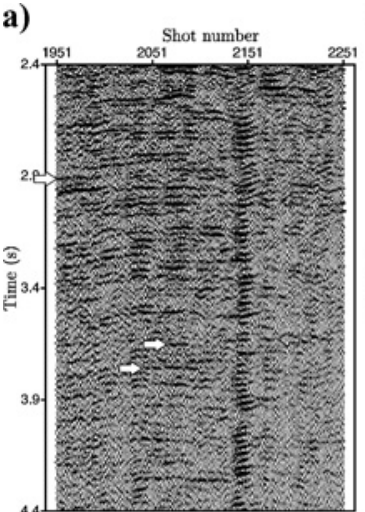

b)

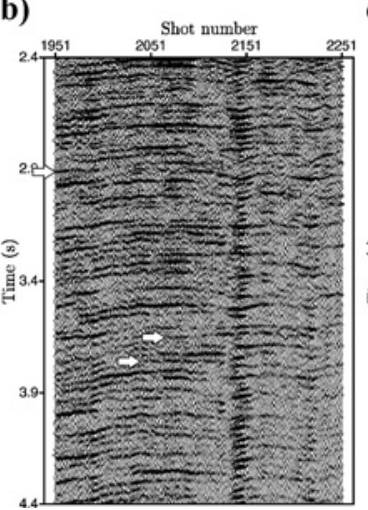

c)

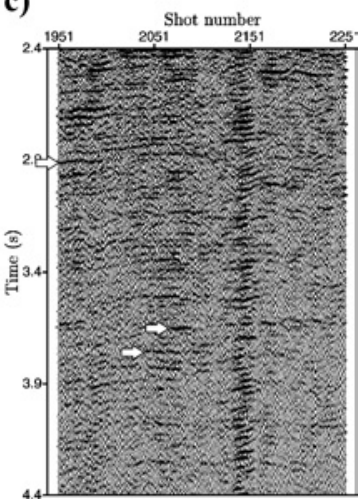

Figure 12. (a) Comparison of the 2D SRME result, (b) the input data commonoffset section, and (c) the 3D MESI result, zoomed in on the right of the input data common-offset section (see Figure 10 for the zoom area). The arrows point to primary reflections that are better preserved by the 3D method than by the 2D method.

the inline offset is $1500 \mathrm{~m}$. The surfacerelated multiple contamination in the common-offset section is clearly observed. The water-layer reverberations and peg-leg multiple reflections especially dominate the section.

Although the lateral subsurface structure is moderate, (local) variations in the medium and consideration of the outer streamer make the prediction of multiples a $3 \mathrm{D}$ problem. The 2D prediction of surface-related multiples for the outer streamer is accomplished by taking the data from the considered streamer as multiple prediction input and operator data (as was proposed by Dragoset et al., 2000). The 2D approach for prediction of surface-related multiples for the common-offset section results in relative time shifts between different types and orders of multiples, which cannot be corrected with one global time shift. The $2 \mathrm{D}$ SRMP result for the zoom area on the right in the common-offset section (indicated with a white frame in Figure 10) is shown in Figure 11a.

The result after the least-squares subtraction of the 2D predicted multiples from the reference common-offset section for the zoom area is shown in Figure 12a. The reduction of multiple energy compared to the reference common-offset section (Figures 11b and $12 \mathrm{~b}$ ) is obvious, although remaining multiple energy can still be observed.

\section{D MPSI for a common-offset section}

For the 3D MPSI method, data from two sail-lines are combined in the calculation of the crossline multiple contributions. Neglecting some spatial shifts, four crossline multiple contributions can be calculated for each multiple-output trace on the outer streamer. The sizes of the neglected spatial shifts are illustrated in Figure 13, in which each arrow beginning at the solid-black source position should connect at the surface contribution location and finish at the receiver position under consideration (the solid-black circle). The resulting crossline multiple-contribution traces for one selected output trace are shown in Figure 14a.

The objective of the sparse inversion is to resolve the stationary multiple contributions (apices of crossline multiplecontribution events). Since 3D effects are caused by geometry rather than geology, most of the surface reflection points are expected to lie within the area of the four crossline multiple contributions. The apex location range is chosen in the same way as the contribution range. The resulting model space is shown in Figure 14b. Note that multiple energy is observed within the complete chosen apex location $\left(y_{0}\right)$ range. For this example, one $q$-value (corresponding to $v_{\text {water }} / 2$ ) was used in the inversion, which shows that the $3 \mathrm{D}$ character of the multiple wavefield is not negligible. Using the estimated model

space, a densely sampled crossline multiple-contribution
Figure 13. The construction of crossline multiple-contribution raypaths for the multiple-output trace of the outer streamer (indicated by the arrow on the right) of the dual-source data set. The source and receiver under consideration are solid black. For the construction of four crossline multiple contributions, data from two sail-lines of the dual-source survey are combined. Note that some spatial shifts are neglected. 
gather can be reconstructed for analysis purposes with the transformation from model space to data space (see Figure 14c). The original four crossline multiple contributions are merged with the reconstructed contributions. As expected from the underdetermined inverse problem, the data fit is
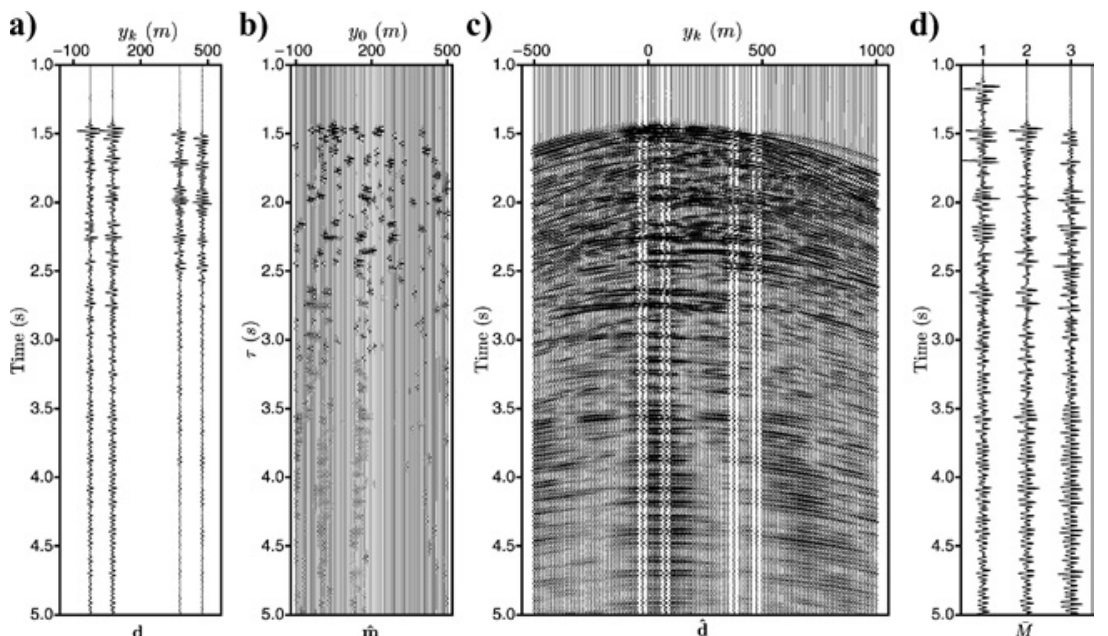

Figure 14. Illustration of the 3D MPSI procedure for the input data commonoffset trace on streamer 8 at shot number 1993. (a) The available four crossline multiple contributions. (b) Final estimate of the model space. (c) The reconstructed crossline multiple contributions using the estimated model space. (d) The input data common-offset data (trace 1), the 3D MPSI result (trace 2), and the 2D SRMP result (trace 3 ).
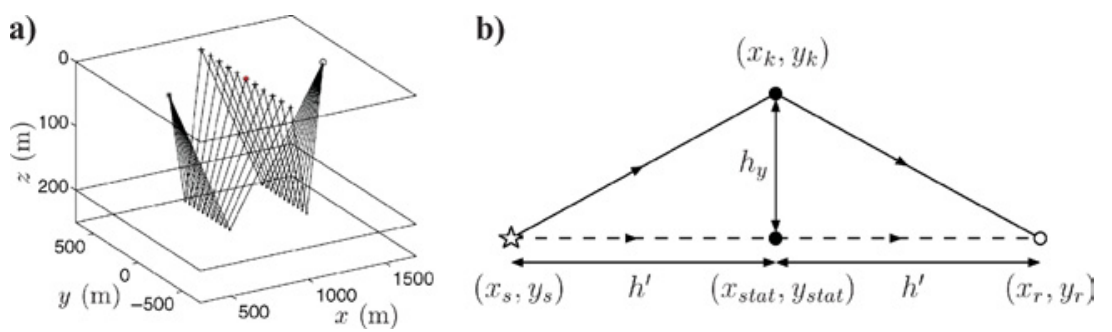

Figure 15. Calculation of crossline multiple-contribution raypaths for a horizontal reflector with $\left(x_{s}, y_{s}\right)=(500,0) \mathrm{m}$ and $\left(x_{r}, y_{r}\right)=(1500,0) \mathrm{m}$. (a) Inline multiplecontribution raypaths around the stationary multiple path. (b) Top view of the 3D construction of multiple-contribution raypaths. The crossline component of the raypaths is $h_{y}$. The stationary multiple raypath is depicted by the dashed line. a)

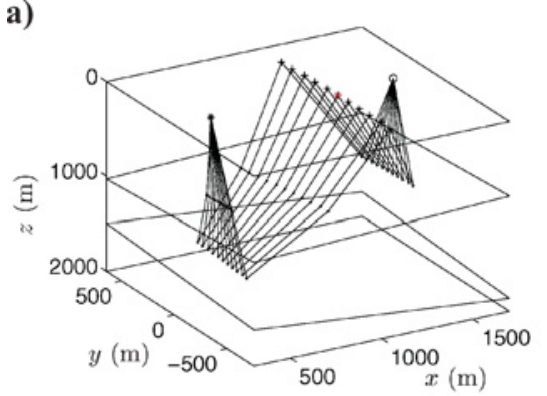

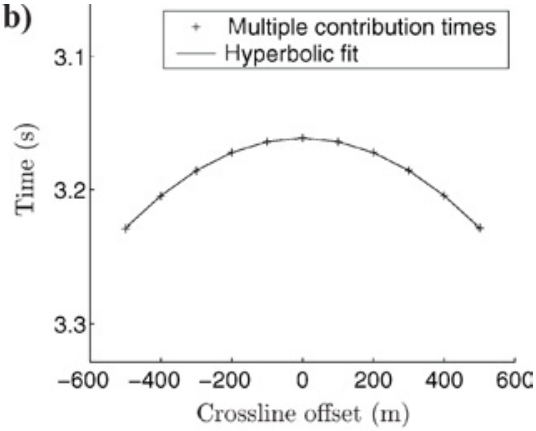

Figure 16. Numerical calculation of multiple-contribution raypaths and traveltimes for the 3D dipping-reflector model with $\left(x_{s}, y_{s}\right)=(500,0) \mathrm{m}$ and $\left(x_{r}, y_{r}\right)$ $=(1500,0) \mathrm{m}$. (a) Crossline multiple-contribution raypaths around the stationary multiple path. (b) Crossline multiple-contribution traveltimes and the leastsquares fitted hyperbolic curve $\left(v^{\prime} \approx 768 \mathrm{~m} / \mathrm{s}\right)$. nearly perfect. Comparison of the four original crossline mulhow the out-of-plane stationary multiple contributions could extracted from the sparse data.

The result of $3 \mathrm{D}$ multiple prediction for the zoom area is shown in Figure 11c. Note that the 3D predicted multiples show a much better resemblance to the input data (see the arrows in Figure $11 \mathrm{~b}$ ) than the $2 \mathrm{D}$ prediction, resulting in an improved multiple-attenuation result (Figure 12c). In the adaptive subtraction stage, the same parameters were used for the $2 \mathrm{D}$ and $3 \mathrm{D}$ results. Primary energy seems to be better preserved in the 3D MESI result compared to the 2D SRME result, as indicated by the arrows in Figure $12 \mathrm{a}-\mathrm{c}$. This conclusion is also drawn by van Borselen and Verschuur (2003), who extend the experiment described in this section to include multiple offsets and compare the 2D SRME results to the 3D MESI results on stack level.

\section{DISCUSSION AND CONCLUSIONS}

In the Fresnel zone reconstruction approach for predicting 3D surface-related multiples, the undersampling of the $3 \mathrm{D}$ data is addressed during 3D multiple prediction. Because of severe undersampling of the wavefield in the crossline direction, the crossline Fresnel summation of multiple contributions cannot be applied. In our approach, the crossline Fresnel summation is replaced with a parametric inversion to extract the stationary contributions from the sparsely sampled crossline multiple contributions that can be calculated with the available input data.

The 3D multiple prediction using the MPSI method consists of three steps: (1) construction of crossline multiple contribution traces from the available data, (2) extraction of $3 \mathrm{D}$ multiple information from the sparsely sampled crossline multiple contributions through a parametric inversion, and (3) translation of the multiple information into the 3D multiple trace.

For the inversion, the crossline multiple contributions are described using three parameters: stationary traveltime, crossline position of the stationary contribution, and the hyperbolic curvature of the crossline multiple-contribution event. We can pose the inverse problem by defining transformation operations from data space (crossline multiple contributions) to model space (containing a range of parameters describing the crossline multiple-contribution events) and back. We find a solution to the ill-posed inverse problem by incorporating a priori 
information in the form of a Cauchy pdf imposed on the model space parameters. The Cauchy pdf drives the solution to sparseness, containing the significant parameters for describing the crossline multiple contributions. Both the range of model space parameters and the sparseness weight influence the accuracy and stability of the inversion and need to be carefully chosen.

The estimated model space contains the 3D multiple information, which can be efficiently translated into the $3 \mathrm{D}$ multiple trace. The 3D multiple information is extracted by applying amplitude and phase corrections to the model space, followed by a summation of the model space traces. The summation process projects the multiple information onto the $3 \mathrm{D}$ multiple trace.

In this article, we have successfully demonstrated the 3D MPSI method on 3D synthetic data sets as well as a 3D marine data set. The 3D MPSI experiments show that the 3D multiple wavefield generated in a subsurface with complex and diffractive 3D structures can be reconstructed (3D synthetic data), but lateral detail in the 3D multiple wavefield also has been resolved (3D marine data set).

Although the 3D MPSI method is a promising alternative to extensive data reconstruction prior to $3 \mathrm{D}$ multiple prediction, some elements of the procedure need more attention: the parameterization of crossline multiple contributions is only approximate, and the sparse inversion is computationally expensive and requires fine-tuning of the model space and inversion parameters. In the examples described in this article, the model space parameters ( $q$-values and $y_{0}$ range) are determined mainly by trial and error. However, analysis of the inline multiple contributions and/or forward modeling could help find the optimum model space and inversion parameters. Another issue that deserves more attention is the fact that the more complex the subsurface, the more crossline multiple contributions are required to resolve the stationary crossline multiple contributions. In addition, application of the 3D MPSI method to current $3 \mathrm{D}$ data sets involves neglect of lateral shifts of assumed coincidental source and receiver lines. Despite these issues, the 3D MPSI method is a promising approach for solving 3D surface-related multiple problems.

\section{ACKNOWLEDGMENTS}

The authors thank Carl Regone from BP Upstream Technology group for providing the 3D synthetic Gulf of Mexico data set and Roald van Borselen (PGS Research) and PGS Geophysical AS and Norske Chevron for providing the 3D marine data set on behalf of the PL259 licence group. The authors also thank the reviewers for their valuable comments and suggestions.

\section{APPENDIX A}

\section{CROSSLINE MULTIPLE-CONTRIBUTION TRAVELTIMES}

In this appendix, crossline multiple-contribution traveltimes are numerically analyzed for two subsurface models: a horizontal reflector model and a model with two 3D dipping plane reflectors.

\section{Horizontal reflector}

For parameterization of the crossline multiple contributions, we express the multiple-contribution traveltimes as a function of the apex position and a curvature parameter. If we consider the crossline multiple contributions for a single horizontal reflector model when both source and receiver are at the same crossline coordinate (Figure 15), the traveltime function of the crossline multiple contributions can be expressed as

$$
t_{\mathrm{MCMO}}\left(t_{0}, h^{\prime}, h_{y}, v\right)=\sqrt{\left(2 t_{0}\right)^{2}+\frac{\left(2 h^{\prime}\right)^{2}+\left(2 h_{y}\right)^{2}}{v^{2}}}
$$

which can be rewritten as the (hyperbolic) function

$$
t_{\mathrm{MCMO}}\left(t_{\text {stat }}, h_{y}, v\right)=\sqrt{t_{\text {stat }}^{2}+\frac{h_{y}^{2}}{(1 / 2 v)^{2}}}
$$

with

$$
t_{\text {stat }}=\sqrt{\left(2 t_{0}\right)^{2}+\frac{h^{\prime 2}}{(1 / 2 v)^{2}}} \quad \text { and } \quad t_{0}=\frac{2 z_{0}}{v}
$$

in which $t_{\text {stat }}$ is the stationary traveltime and $t_{0}$ is the traveltime of the vertical part of the raypath (once down and up, $z_{0}$ represents the depth of the reflector), and $h^{\prime}$ represents the inline distance from source or receiver to the stationary reflection point. The hyperbolic moveout is dependent on the crossline component of the offset from the stationary reflection $\left(h_{y}=y_{\text {stat }}-y_{k}\right)$, the traveltime of the stationary contribution $t_{\text {stat }}$, and the velocity of the medium $v$.

If a crossline offset between the source and receiver is introduced, the traveltime function of the crossline multiple contributions deviates from the hyperbolic expression. For situations with varying reflector depth and crossline sourcereceiver offset, we numerically analyze the traveltimes of the crossline multiple contributions. We calculate the crossline multiple contributions and fit a hyperbolic function to the traveltimes in a least-squares sense, resulting in a curvature that corresponds to an effective velocity $v^{\prime}$ (which in the previous situation would relate to the medium velocity as $v^{\prime}=v / 2$ ). The effective velocities are displayed in Table 1 as a function of reflector depth and source and receiver coordinates. From the

Table 1. Hyperbolic curve fitting through the crossline multiple-contribution traveltimes for the first-order multiple in horizontal reflector models. The curvature determines the effective velocity.

\begin{tabular}{lllr}
\hline$z_{\text {refl }}(\mathrm{m})$ & $\left(x_{s}, y_{s}\right)(\mathrm{m})$ & $\left(x_{r}, y_{r}\right)(\mathrm{m})$ & $v^{\prime}(\mathrm{m} / \mathrm{s})$ \\
\hline 200 & $(500,-100)$ & $(1500,100)$ & 759 \\
200 & $(500,-200)$ & $(1500,200)$ & 787 \\
200 & $(500,-500)$ & $(1500,500)$ & 1019 \\
500 & $(500,-100)$ & $(1500,100)$ & 753 \\
500 & $(500,-200)$ & $(1500,200)$ & 761 \\
500 & $(500,-500)$ & $(1500,500)$ & 824 \\
1000 & $(500,-100)$ & $(1500,100)$ & 751 \\
1000 & $(500,-200)$ & $(1500,200)$ & 754 \\
1000 & $(500,-500)$ & $(1500,500)$ & 771 \\
\hline
\end{tabular}


table, it is clear that the effective velocity approaches half the medium velocity when the ratio of (absolute) crossline offset between source and receiver $\left(y_{s}-y_{r}\right)$ and depth of the reflector $\left(z_{\text {refl }}\right)$ is small.

\section{D dipping reflectors}

The second model used in analysis of the crossline multiplecontribution behavior is the model with two 3D dipping reflectors, illustrated in this article (Figures 1 to 3 ). The model is displayed in Figure 16a. We analyze the behavior of the crossline multiple-contribution traveltimes for four types of first-order surface multiples and two second-order waterbottom multiples.

Figure 16a shows the crossline multiple-contribution raypaths for the receiver side peg-leg multiple, coded as 0121010. (In the coding, the numbers indicate, from left to right, the order in which the ray has reflected at or passed the reflectors. The surface is represented by 0 .) The stationary reflection at the surface is at $\left(x_{\text {stat }}, y_{\text {stat }}\right)=(1163,-57) \mathrm{m}$. The crossline multiple-contribution traveltimes corresponding to the raypaths are shown in Figure 16b. The effective moveout velocity of the hyperbolic curve fitted in a least-squares sense to the traveltimes is $v^{\prime} \approx 768 \mathrm{~m} / \mathrm{s}$.

Results of the analysis for different types of surface multiples are summarized in Table 2. For the second-order waterbottom multiples, the analyzed surface reflection is underlined. Crossline multiple contributions are analyzed for two multiple trace locations, of which one has a crossline offset between the source and receiver.

The main conclusion to be drawn from this analysis is that the introduction of a relatively small crossline offset between the source and receiver mainly affects the stationary reflection position at the surface and barely affects the effective velocity of the fitted hyperbolic curve. The effective velocity of the crossline multiple-contribution moveout is nearly half the water velocity for most of the multiple raypaths that spend most of their traveltimes in the water column. The (mean) medium velocity is the main factor that determines the effective velocity for the 3D models used in this analysis.

Table 2. Hyperbolic curve fitting through the crossline multiplecontribution traveltimes for different types of multiples in the 3D dipping-reflector model. The curvature determines the effective velocity. The multiple type is described by the subsequent points of contact of the multiple raypath with the reflectors (reflection or transmission).

\begin{tabular}{lcccr}
\hline $\begin{array}{l}\text { Multiple } \\
\text { type }\end{array}$ & $\begin{array}{c}\left(x_{s}, y_{s}\right) \\
(\mathrm{m})\end{array}$ & $\begin{array}{c}\left(x_{r}, y_{r}\right) \\
(\mathrm{m})\end{array}$ & $\begin{array}{c}v^{\prime} \\
(\mathrm{m} / \mathrm{s})\end{array}$ & $\begin{array}{r}\left(x_{\text {stat }}, y_{\text {stat }}\right) \\
(\mathrm{m})\end{array}$ \\
\hline 01010 & $(500,0)$ & $(1500,0)$ & 749 & $(1181,-157)$ \\
0101210 & $(500,0)$ & $(1500,0)$ & 762 & $(799,-57)$ \\
0121010 & $(500,0)$ & $(1500,0)$ & 768 & $(1163,-57)$ \\
012101210 & $(500,0)$ & $(1500,0)$ & 842 & $(581,154)$ \\
0101010 & $(500,0)$ & $(1500,0)$ & 703 & $(1489,-308)$ \\
0101010 & $(500,0)$ & $(1500,0)$ & 724 & $(1180,-318)$ \\
01010 & $(500,0)$ & $(1500,-400)$ & 752 & $(1187,-366)$ \\
0101210 & $(500,0)$ & $(1500,-400)$ & 765 & $(793,-184)$ \\
0121010 & $(500,0)$ & $(1500,-400)$ & 765 & $(1177,-333)$ \\
012101210 & $(500,0)$ & $(1500,-400)$ & 847 & $(570,-30)$ \\
0101010 & $(500,0)$ & $(1500,-400)$ & 696 & $(1490,-576)$ \\
0101010 & $(500,0)$ & $(1500,-400)$ & 734 & $(1185,-462)$ \\
\hline
\end{tabular}

\section{REFERENCES}

Anderson, J. E., 1996, Imaging in transversely isotropic media with a vertical symmetry axis: Ph.D. thesis, Colorado School of Mines.

Berkhout, A. J., 1982, Seismic migration, imaging of acoustic energy by wave field extrapolation, A: Theoretical aspect: Elsevier Science Publishing Co., Inc.

Berkhout, A. J., and D. J., Verschuur, 1997, Estimation of multiple scattering by iterative inversion, part I: Theory: Geophysics, 62, 1586-1595.

Biersteker, J., 2001, MAGIC: Shell's surface multiple attenuation technique: 71st Annual International Meeting, SEG, Expanded Abstracts, 1301-1304.

Bleistein, N., 1984, Mathematical methods for wave phenomena: Academic Press, Inc.

Dragoset, W. H., and Z̆. Jeričević, 1998, Some remarks on surface multiple attenuation: Geophysics, 63, 772-289.

Dragoset, W. H., J. Bell, R. Vauthrin, and D. Pattberg, 2000, Surface multiple attenuation: A 3-D example: 70th Annual International Meeting, SEG, Expanded Abstracts, 1957-1960.

Etgen, J. T., and C. J. Regone, 1998, Strike shooting, dip shooting, widepatch shooting - Does prestack depth migration care? A model study: 68th Annual International Meeting SEG, Expanded Abstracts, 66-69.

Ikelle, L. T., and S. Yoo, 2000, An analysis of 2D and 3D inverse scattering multiple attenuation: 70th Annual International Meeting, SEG, Expanded Abstracts, 1973-1976.

Kabir, M. M. N., and D. J. Verschuur, 1995, Restoration of missing offsets by parabolic Radom transform: Geophysical Prospecting, 43, 347-368.

Kinneging, N. A., V. Budejicky, C. P. A. Wapenaar, and A. J. Berkhout, 1989, Efficient 2D and 3D shot record redatuming: Geophysical Prospecting, 37, 493-530.

Matson, K. H., and D. Corrigan, 2000, A 2.5D method for attenuating free-surface multiples based on inverse scattering series: $32 \mathrm{nd}$ Annual International Meeting, Offshore Technology Conference, Proceedings, 12046.

Nekut, A. G., 1998, 3D surface-related multiple prediction: 68th Annual International Meeting, SEG, Expanded Abstracts, 15111544.

Ross, W. S., Y. Yu, and F. A. Gasparotto, 1999, Traveltime prediction and suppression of 3-D multiples: Geophysics, 64, 261-277.

Sacchi, M. D., and T. J. Ulrych, 1995, High-resolution velocity gathers and offset space reconstruction: Geophysics, 60, 1169-1177.

Sacchi, M. D., T. J. Ulrych, and C. J. Walker, 1998, Interpolation and extrapolation using a high-resolution discrete Fourier transform: IEEE Transactions on Signal Processing, 46, 31-38.

Shewchuk, J. R., 1994, An introduction to the Conjugate Gradient method without the agonizing pain: School of Computer Science, Carnegie Mellon University.

Sun, Y., 1999, Anti-aliasing multiple prediction beyond 2-D: 69th Annual International Meeting, SEG, Expanded Abstracts, 13381341.

Thorson, J. R., and J. F. Claerbout, 1985, Velocity-stack and slantstack stochastic inversion: Geophysics, 50, 2727-2741.

van Borselen, R. G., and D. J. Verschuur, 2003, Optimization of marine data acquisition for the application of 3D SRME: 73rd Annual International Meeting, SEG, Expanded Abstracts, 1965-1968.

van Dedem, E. J., 2002, 3D surface-related multiple prediction: Ph.D. thesis, Delft University of Technology.

Verschuur, D. J., 1991, Surface-related multiple elimination, an inversion approach: Ph.D. thesis, Delft University of Technology.

Verschuur, D. J., and A. J. Berkhout, 1997, Estimation of multiple scattering by iterative inversion, part II: Practical aspects and examples: Geophysics, 62, 15961611.

Verschuur, D. J., A. J. Berkhout, and C. P. A. Wapenaar, 1992, Adaptive surface-related multiple elimination: Geophysics, 57, 1166-1177.

Wapenaar, C. P. A., 1992, The stationary phase method and its application in seismics and acoustics: Lecture notes: Akoestische Beeldvorming, Delft University of Technology.

Weglein, A. B., 1999, Multiple attenuation: An overview of recent advances and the road ahead: The Leading Edge, 18, 40-44.

Zwartjes, P. M., and A. J. W. Duijndam, 2000, Optimizing reconstruction for sparse spatial sampling: 70th Annual International Meeting, SEG, Expanded Abstracts, 2162-2165. 